für Volks- und Landesnamen; man wird eine/Als Hauptstadt des Zaghāwa-Reiches wird dār- und eine $b a-Z$ Zne unterscheiden können), M̄ānān genannt, daneben kommt aber auch s. Nachtigal 3, 199, auch 35. Wiederum zu die Stadt Zaghăwa vor. Diese Orte werden trennen ist das $k \bar{u} k a=$ Affenbrotbaum, das Idrisi $12 \mathrm{f}$. in Beziehung gesetzt zu Bilma, verwandt wurde zur Bezeichnung der neuen zu Enǧimī, zu Tãğo, aber nicht zu Qórān sind die Tubu (oder Tedā) Wadaïs (Nachtigal 3, 212), von denen ein bedeutender Teil im Baḥr alghazāl lebt; das würde dafür sprechen, den نهر in diesem Flusse zu sehen, der von dem Gebirge hinter Borku (von Burgū zu trenuen!) herkommt, und in diesem Gebirge, bezw. in dem ganzen Gebirgslande von Tibesti hätten wir dann die $\ddot{g} i b \bar{a} l$ lünija zu suchen, die Idrisi erwähnt $(38,11)$, und die ich als die "Libyschen Berge" deuten möchte. Sehen wir den نهر in dem Batha, so haben wir auch die Herkunft ans einem Gebirge; es ist dann an das auf den Karten als "Targa-Gebirge" an der Grenze von Wadaï und "Dār-Fōr eingetragene Gebirge (Dschebel Marra auf der Karte Nachtigals) zu denken, dessen Fortsetzung bis zum Nil weiter nach Osten hin sich leicht vorstellen liess!.

Es wird sich wohl nie mit vollkommener Sicherheit entscheiden lassen, an welchen der beiden Flussläufe und an welches der beiden Gebirge die arabischen Geographen gedacht haben, schon deshalb nicht, weil die Nachrichten die sie darüber besassen, sehr unbestimmt waren, und weil sie von der Situation nur eine ganz unklare Vorstellung hatten. Das aber ist, denke ich, durch eine schlüssige Argumentation nachgewiesen, dass Kaukau-Kügu weit im Osten lag und in Wadaï zu suchen ist. Dieses Kūgū floss den arabischen Geographen mit dem äbnlich klingenden Küga zusammen, und es entstanden Mischnachrichten, die uns vor unlösbare Rätsel stellen.

Nun noch ein Moment, das ich nicht als Hauptstütze betrachte, das aber, wenn einmal die Gleichung Kaukau-Kugū-Wadaï gesichert ist, besondere Bedeutung erhält. Kaukau-Kūgū wird in Beziehung gebracht zu dem Reiche Zaghāwa in dem Sinne, dass die Zaghāwa Nachbarn des Landes Kūgū sind. Lassen sich die Zaghāwa als Nachbarn von Wadaï erweisen, so fällt wohl auf das, was die arabischen Autoren von ihnen berichten, einiges Licht.

1 Das Bild des Sudan ist noch bis zum Ende des 18. Jahrhunderts arg verschoben. Auf den Karten Rennells von 1790 und 1799 ist die Entfernung zwischen Nil und Tschadsee viel zu kurz; Bornu und Kanem liegen östlich von ihm, Bornu an dem "Wad-el-Gazel" (Bahr alghazäl), das mit dem Nil in Verbindung steht, zuglejch aber gich in den Tschadsee ergiesst. Das ist dieselbe alte Vorstellung, die in den arabischen Texten zum Ausdrucke kommt. dieses dem Verfasser mit Küga zusamenfliesst und zu weit westlich gedacht ist. Ist auf die Nachricht Jaqut 4, 230 etwas za geben, dass die Kauwär-Oasen einen Sultan haben, der dem König der Zaghāwa untertan ist, so dürfen wir annebmen, dass das Reich Zagbāwa sich nördlich von Kügĩ-Wadaï und auch nördlich von Kănem hinzog, mithin sein Hauptgebiet in der Steppe hatte, wie es sich denn uns in den Quellen als ein Nomadenreich darstellt. Dass Zaghāwa Kānem beherrscht babe, dafür liegen Beweise nicht vor. In keinem Falle kann man sagen (Becker a. a. O. 169), dass das grosse heidnische Reich der Zaghāwa, das vor der Gründung von Känem-Bornu bestand, ,sich von Nubien bis an den Niger erstreckte". Diese Konstruktion stützt sich auf die in den Quellen berichtete Nachbarschaft Zaghāwas mit und die Annahme, dieses كوكو sei Gao am Niger. Diese Annahme ist aber unrichtig.

\title{
Die Kaspierkönige sind nicht arischer Herkunft.
}

Von Ferdinand Bork.

Für gewisse Kreise unserer Altorientalisten und Historiker hat der Fund von Boghazköi, wie es scheint, eine wabre Umwälzung aller Vorstellungen über die Vorgeschichte der Arier in Asien und die Völkergruppierungen im alten Vorderasien bedeutet: für diejenigen nämlich, die trotz Awesta und Weda die Frage nach Ariern vor 850 ganz ausşchalteten und nicht minder für die Awesta- und Wedaforscher, die nicht zu bewegen waren, sich um die Keilschriftforschung $z u$ kümmern.

Seitdem nun die Inder - denn um diese handelt es sich, nicht um Indo-Iranier vor der Trensung - um 1400 in Armenien nachgewiesen sind, wird der Gedanke wieder nahe gelegt, ob nicht auch die Kaspier, deren Gebiet die Inder also durchzogen haben müssen, Arier gewesen sein möchten. Und da diese offenbar die Kassi der Assyrer sind, so ist es zweifellos berechtigt, wenn man sich nach Spuren indischer Sprache in unseren Keilschrifttexten umsieht; ebenso berechtigt wäre es freilich auch, im Weda die Spuren kaspischer Sprache, z. B. in Namen von Göttern, Menschen, Völkern usw. zu suchen, dèn es spricht nichts dafür, dass die Kaspier Inder wären, wohl aber sind die Kassi Kaukasier, und somit auch die $\boldsymbol{K} \alpha \sigma \pi \iota 0 \iota$. 
Dhorme hat in seinem Aufsatze "Les Aryens avant Cyrus" (Conférences de Saint-Etienne 1910-11) den Nachweis versucht, dass das Pantheon der Kaspier arische Bestandteile enthalte, und schliesst daraus auf eine arische Herrenschicht in der Bevölkerung. In der Revue Biblique 1911 VIII 2 bringt er für seine Vermutung einen neuen Beleg bei, den Namen Kurin-da-ar, den er auf einem babylonischen Täfelchen gefunden hat. Er trägt kein Bedenken, den Bestandteil indar mit arischem Indra zu vergleichen und verweist auf seine anderen Gleichungen Suriaš oder Šuriaš = suriya "Sonne", Buriaš $=$ Bogras. In dem früheren Aúfsatze hatte er den kaspischen Namenteil marataš $(=$ Weltschöpfer) mit indischem marút zu verbinden gesucht.

Es kostet keine Anstrengung, solche Gleichungen auszusprechen, und wir möchten uns dagegen verwahren, dass das ein wissenschaftliches Verdienst von irgend welchem Werte wäre. In solchem Falle, wo einem jeden sich die Gleichung aufdrängt, ist es wertlos, sie auszusprechen, ohne zu erwägen, ob der Anklang nicht etwa auf Zufall beruhe. Dies ist durchaus möglich, und es muss von Fall zu Fall untersucht werden. Ist aber die Gleichung als solche richtig, dann muss eben auch gezeigt und begrïndet werden, wer der entlehnende Teil war. Im anderen Falle bedeutet ein etwaiges "Rechtbehaltenhaben" einen Lotteriegewinn ohne jedes Verdienst des Spielers.

Nehmen wir die Gleichung als richtig an, dann ist zunächst festzustellen, dass weder für Bo@zas noch für Marut eine arische Herkunft erweisbar oder auch nur wahrscheinlich ist; aber auch das indische Wort Sürjas ist nicht einmal den Iraniern bekannt. Die Etymologie von der $\sqrt{\text { swar }}$ kann also leicht eine nachmalige sein.

Liegt in diesen drei Fällen eine Entlehnung vor, dann sind doch wohl die Inder der entlehnende Teil, nicht die Kaspier! Im Kaspischen sind Namen wie suriaš, buriaš analoge Bildungen von Stämmen, die wir als Suri, Buri anzusetzen haben, adjektivische Bildungen wie Sassiaš, Araziaš, Dupliaš u. a. m.; maru-taš aber ist Verbalform mit vorhergehendem Objekte. Gibt Dhorme nun einen Kur-in-da-ar bekannt, wo wir bei den Kaspier-Königen Namen wie Karindaš, Kurgalzu antreffen, und das $\breve{s}$ stark im Verdachte steht, ein . $\check{r}$ zu sein, dann wollen wir lieber abwarten, ob sich ein Kur-ilu-In$d a$-ar finden werde, und bis dahin im in das Objektivum, in dar die Verbalform eines echt kaspischen Namens sehen! Von indischem oder überhaupt arischen Einflusse auf die Kassi
Kann nach dem heute vorliegendem Stoffe nur reden, wer den Dingen ganz ferne steht, und nichts berechtigt uns zu der Vermutung, dass die Könige der Kassi arischer Herkunft gewesen wären.

\section{Bemerkungen zu den Asarhaddoninschriften.}

Von Brano Meissner.

\section{1. amutu.}

Asarbaddon Schw. St. III 24 (I R. 49) ist eine alte crux interpretum. Es heisst dort Kol. III $15 \mathrm{ff}$. (vgl. MeIssNer-Rost, Bauinschr. Asarh. in BA III 220): Um selbiges Werk auszuführen, habe ich mich der Entscheidung des Samas, Adad, des Oberrichters Merodach, der Götter, meiner Herren, gebeugt und sprach vor ihnen. Durch das Wahrsagegerät der Seher geschahen vertrauenerweckende Vorzeichen, Babylon zu bauen, Esagila zu erneuern liessen sie (die Götter) aufschreiben $T+1$. Ein genauer Paralleltext ist K. 192 Rs. 10 (s. ib. 244), nur steht dort $i$ išsa-aš-ti-ru Zeichen hatte JäGGR BA I 448 gelesen ana iá-tú; indes wäre diese Schreibung doch sehr sonderbar, da als ia ja wohl vorkommt, in der Form iâti, iâssi diese Schreibung aber m. W. noch nicht beobachtet ist. Nicht besser war unser (MerssNer-RosT) Vorschlag, (ana) mu-hiš zu lesen; denn muhu für muhhhu wäre in dieser Zeit ungewöhnlich, und dann hat das Suffix šu keine Beziehung.

In den neuen in Assur gefundenen Inschriften berichtet nun Asarhaddon über seine Bauten ganz ähnlich mit folgenden Worten (MESSERSCHMIDT, Keilschrifttexte aus Assur histor. Inhalts I Nr.51, III 1 ff.): [ina makalti barûtiti] i-pu-lu-ni-ma šá e-piš

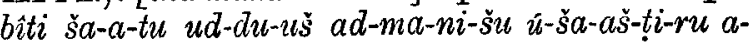
mu-tum $=$ [Durch das Wahrsagegerät der Seher] haben sie (die Götter) mir geantwortet und, selbiges Haus zu bauen und sein Gebäude zu erneuern, ein amutu aufschreiben lassen. Aus dieser Stelle sieht man, dass die Lesung $m u$ $h i \check{s}$ jedenfalls aufzugeben ist. Falls die neue Edition, aus der allerdings nicht zu ersehen ist, inwieweit sie auf dem Studium des Originals beruht, richtig ist, hätten wir anzunehmen, dass I R. 49 für Y wohl If einzusetzen wäre und dass K. 192 entweder $Y Y$ ausgefallen ist oder dass neben amutu auch die Form mutu existiert. amutu bedeutet, wie schon Desirzsch $\mathrm{HW} 81 \mathrm{~b}$ vermutete, einen Körperteil. MVAG 1905, 249 wies ich dann nach, dass amutu ein Synonym von takâltı ist und "Eingeweide" oder einen bestimmten Teil der Eingeweide, etwa den Magen bedeute; vgl. auch FRANK, Stud. z. babyl. 